\title{
Reversing Phenotype from Epithelial-Mesenchymal Transition (EMT) to Mesenchymal-Epithelial Transition (MET) with Eribulin: Case Report
}

\section{Jai Bhagwan Sharma*}

Medical Oncology, H.O.D. and Senior Consultant, Action Cancer Hospital, New Delhi, India

*Corresponding Author: Jai Bhagwan Sharma, Medical Oncology, H.O.D. and Senior Consultant, Action Cancer Hospital, New Delhi, India.

DOI: $10.31080 /$ ASCB.2020.04.0248
Received: July 22, 2020

Published: July 30, 2020

(C) All rights are reserved by Jai Bhagwan

Sharma.

\section{Abstract}

Breast cancer remains the most frequently diagnosed cancer in women and, despite significant improvements in treatment, it remains the second leading cause of death from cancer.

Keywords: Epithelial-Mesenchymal Transition (EMT); Mesenchymal-Epithelial Transition (MET); Eribulin

\section{Introduction}

Breast cancer remains the most frequently diagnosed cancer in women and, despite significant improvements in treatment, it remains the second leading cause of death from cancer. Like any other solid tumor, it can be located or metastasized in the lymph node and in another distant organ. Once breast cancer metastasizes, the goal of the treatment changes from curative to the patient's prolonged survival and improved quality of life [1].

The therapeutic approach to metastasis is often based on the status of important markers, such as hormone receptors or epidermal growth factor receptor 2 (HER2) which is the most powerful predictive biomarker for determining the efficacy of anti-HER2 therapy [2]. The primary means to evaluate the patient is immunohistochemistry to detect the expression of the HER2 protein in the membranes of cancer cells [3]. Patients with positive HER2 disease and/or hormone receptor have improved their survival due to new treatment options, while survival rates remain low in patients with triple negative breast cancer [4].

Treatment strategies for patients with advanced breast cancer (ABC) positive for human epidermal growth factor (HER2) have increased significantly in recent decades. The results in patients with ABC HER2 positive were significantly improved with the introduction of multiple anti-HER2 therapies, including trastuzumab, pertuzumab, lapatinib and ado-trastuzumab emtansine (T-DM1) [5]. Currently, the median survival of patients treated with these anti-HER2 treatments is greater than 5 years, compared to only 1.5 years in the absence of several anti-HER2 treatments [6].

The treatment options in metastatic breast cancer $(\mathrm{mBC})$ are based mainly on the biology of the primary tumor [7]. The effective treatment options with proven survival benefits are necessary for these patients. However, it is often difficult for clinicians to evidence-based judgements regarding the most effective treatment for such patients [8].

Research conducted in recent decades has provided a better understanding of the complex molecular heterogeneity of this malignancy. One such finding was the identification of the HER2 gene, which encodes a tyrosine kinase receptor, a potent mediator of cell growth and proliferation in normal and malignant epithelial cells. The amplification of this gene was observed in almost $25 \%$ of breast cancers and has been shown to be a driver of tumor biology [9]. 
Recent reports had suggested that eribulin is a candidate of choice for the treatment of HER2 positive breast cancer in patients who have been previously treated with taxanes and anthracyclines [10]. Eribulin mesylate is a microtubule inhibitor that is a synthetic analogue of haliclondrine B modified by structural modifications. It binds only to the growth of positive ends, inhibiting the growth phase of the microtubules without affecting the shortening phase and causing the sequestration of tubulin in non-productive aggregates. This unique mechanism of action based on tubulin may explain how eribulin is able to overcome resistance to taxanes and may have a broader clinical effect [11].

First, it promotes the relaxation of the centromere axis without affecting the speed of stretching.

Then, eribulin sequesters tubulin into non-functional aggregates that can compete with soluble tubulin for addition at the end of growing microtubules. Due to its effects on the microtubule, eribulin causes the arrest of the G2/M phase of the cell cycle and prolonged mitotic blockade, with a marked alteration of the architecture of the normal mitotic spindle, which leads to the activation of the apoptotic cascade and cell death [12].

In addition to having a primary anticancer mechanism associated with conventional antimitotic effects, eribulin also makes residual tumors less aggressive and less likely to metastasize by triggering the transition from epithelial to mesenchymal phenotypes by reversing EMT status to MET [13].

It has also been clinically observed that eribulin suppresses the incidence of new metastases, which provides a greater survival benefit for patients, even under conditions conducive to the evolution of the primary tumor and the pre-existing metastatic tumor. More recently, EMT has been shown to be a key step in the promotion of metastasis in many types of cancer [14].

Herein we report the case of a patient with heavily pre-treated metastatic breast cancer who received eribulin in the late-line metastatic breast cancer setting and showed suppression of further spread of metastasis leading to enhancing the efficacy of the later drug.

\section{Case Presentation}

In October 2007, a 53 year old female patient was diagnosed with carcinoma of left breast. The patient had been submitted to modified radical mastectomy (MRM) on left side in January 2007, with histopathology for infiltrating ductal carcinoma, highly positive estrogen receptor, progestin receptor, and HER-2-NEU-1+. The pathological tumor node metastasis (pTNM) stage was pT2 pN1 M0. The patient received 4 cycles of chemotherapy with anthracycline and paclitaxel each along with adjuvant radiotherapy. She was put on oral therapy with anastrozole $1 \mathrm{mg}$ for up to 6 years.

In the following 7 years, the patient was clinically node negative. In until April 2014, when the patient complained of lower back pain, lower limb pain and the PET-CT scan revealed bone metastasis. She was put on everolimus plus exemestane (reduction in dose was done due to poor tolerance) with zoledronic acid.

In 2015, there was progression of disease effecting liver and bone received adjuvant chemotherapy with 6 cycles of Nab-paclitaxel and carboplatin followed by zoledronic acid. After regular follow-up, there was no sign of recurrence and was maintained on hormonal therapy of fulvestrant with denosumab from 2016.

In 2017, there were metabolically active skeletal and hepatic metastasis during CT scan evaluation signifying as disease progression due to new development of abdominal lymph nodes affecting bone and liver. She was treated with palbociclib and letrozole for 3 months but still had disease progression. Subsequently, she underwent chemotherapy in metastatic set up with eribulin (dose of $1.4 \mathrm{mg} / \mathrm{m}^{2}$ ) along with zoledronic acid. After 3 cycles of eribulin, the patient showed partial response and the treatment was continued for another 3 cycles with zoledronic acid (D1 of cycle 4 and cycle 6). The response obtained disease progression was a stable disease with complete resolution.

Later, the patient was put on capecitabine. The revaluation performed after 9 doses of therapy in April 2018 revealed non FDG avid few hypodense lesions liver-likely healed lesions, no evidence of metabolically active lesions in the post po left chest wall region or elsewhere in the visualized region of the body. The patient was put on maintenance therapy with denosumab at a dose of $120 \mathrm{mg}$ and was admitted for $21^{\text {st }}$ dose on January 2019 with other supportive care.

\section{Discussion}

Despite the wide pharmacological armamentarium available for the treatment of MBC, long-term control of the disease remains difficult, especially in highly pre-treated patients [15]. In the case of HER2 positive breast cancer, chemotherapy is a key backbone with 
which HER2-based therapy is combined as separate agents administered simultaneously or, more recently, the use of chemotherapy conjugated to a HER2 antibody [16].

Eribulin, is therefore, part of broad list of potential therapeutic options, has a mechanism of action distinct from that of other tubulin-targeting agents, with proven tolerance and efficacious in this hard-to-treat breast cancer population because its distinct mechanism of action may allow it to be active in patients whose disease is refractory to tubulin-targeting agents [17]. The recent studies, eribulin has been shown to exert non-mitotic mechanisms in three categories: reversion of the epithelial-mesenchymal transition (EMT), effects on tumor vascular remodelling and perfusion, and decreased capacity for migration and invasion. These non-mitotic effects occur in the surviving residual tumor, including its component tumor cells, as a result of the antimitotic cytotoxic activity of eribulin [18]. In the preclinical xenograft models of breast cancer in humans, eribulin caused the vascular remodeling of the tumor, which resulted in a greater perfusion of the tumor cores [19]. The hypoxia effect was observed which is associated with increased numbers of micro vessels in tumors that had been exposed to a single dose of eribulin approximately 1 week earlier. There was enhanced antitumor activity of capacetabine in the MDA-MB-231 xenograft model due to prior treatment with eribulin [20]. These findings suggest that eribulin-induced vascular remodeling leads to changes in the tumor microenvironment that reduce or eliminate hypoxia-driven growth aggressiveness and increase exposure of subsequent treatments [21].

The patient in the case described, with extensive treatment experience in the metastatic setting and few new treatment options, was able to derive clinical benefit from eribulin. According to the working hypothesis that, after eribulin, residual tumors are phenotypically different and probably less aggressive. Due to induced epithelial-mesenchymal transition, eribulin could also influence cancer immunoediting [22].

\section{Conclusion}

Effective treatment of patient with highly pretreated metastatic breast cancer remains a challenge. However, new therapeutic options, the introduction of eribulin into the therapeutic arsenal, reinforce the hope that the prolongation of survival, as well as the quality of life maintained/improved, is a feasible goal for cancer patients, as in the current case study.
Bibliography

1. Ankur S. "Triple Negative Breast Cancer: A Unique Type of Breast Cancer". Reconstructive Surgery and Anaplastology 6.1 (2017).

2. Montemurro F., et al. "Human epidermal growth factor receptor 2 (HER2)-positive and hormone receptor-positive breast cancer: new insights into molecular interactions and clinical implications". Annals of Oncology 2013 24: 2715-2724.

3. Maria CAS., et al. "Management of metastatic HER2-positive breast cancer: Where are we and where do we go from here?" Oncology 30.2 (2016): 148-155.

4. Shreshtha M., et al. "Epidemiology of breast cancer in Indian women”. Asia-Pacific Journal of Clinical Oncology 13 (2017): 289-295.

5. Larionov AA. "Current Therapies for Human Epidermal Growth Factor Receptor 2-positive metastatic Breast Cancer". Frontiers in Oncology 8 (2018): 89.

6. Gurprataap SS., et al. "Prevalence of Triple-Negative Breast Cancer in India: Systematic Review and Meta-Analysis". Journal of Global Oncology 2.6 (2016): 412-421.

7. Andre F and Zielinski CC. "Optimal strategies for the treatment of metastatic triple-negative breast cancer with currently approved agents". Annals of Oncology 23.6 (2012): 46-51.

8. Twelves C., et al. "Efficacy of eribulin in women with metastatic cancer: a pooled analysis of two phase 3 studies". Breast Cancer Research and Treatment 148 (2014): 553-561.

9. Drakaki A and Sara AH. "HER2-Positive Breast Cancer: Update on New and Emerging Agents". The American Journal of Hematology/Oncology 11.4 (2015): 17-23.

10. Araki K. "First report of Eribulin in combination with pertuzumab and trastuzumab for advanced HER2-positive breast cancer". The Breast 35 (2017): 78-84.

11. Gorouhi F and Gluck S. "Eribulin monotherapy in a patient with heavily pretreated metastatic breast cancer: Case study and review of the literature". Journal of Solid Tumors 3.1 (2013).

12. Menis J and Twelves C. "Eribulin (Halaven): a new, effective treatment for women with heavily pretreated metastatic breast cancer". Breast Cancer: Targets and Therapy 3 (2013): 101-111. 
13. Goto W., et al. "Eribulin Promotes Antitumor Immune Responses in Patients with Locally Advanced or Metastatic Breast Cancer". Anticancer Research 38 (2018): 2929-2938.

14. Yoshida T., et al. "Eribulin mesilate suppresses experimental metastasis of breast cancer cells by reversing phenotype from epithelial-mesenchymal transition (EMT) to mesenchymalepithelial transition (MET) states". British Journal of Cancer 110 (2014): 1497-1505.

15. Medici M and Fossile E. "Long-Term Response with Eribulin Mesylate in a Breast Cancer Patient: A Case Report". Oncology 94.1 (2018): 3-5.

16. Sallinas CA., et al. "Complete Response of Metastatic Androgen Receptor-Positive Breast Cancer to Bicalutamide: Case Report and Review of the Literature". Journal of Clinical Oncology 32.4 (2014): 21-24.

17. Jordan MA., et al. "The primary antimitotic mechanism of action of the synthetic halichondrin E7389 is suppression of microtubule growth". Molecular Cancer Therapeutics 4.7 (2005): 1086-1095.

18. Ramaswami R., et al. "Activity of eribulin mesylate in heavily pretreated breast cancer granted access via the Cancer Drugs Fund". Future Oncology 10.3 (2014): 363-376.

19. Cortes J., et al. "Multiple modes of action of eribulin mesylate: Emerging data and clinical implications". Cancer Treatment Reviews 70 (2018): 190-198.

20. Yoshida T., et al. "Eribulin mesilate suppresses experimental metastasis of breast cancer cells by reversing phenotype from epithelial-mesenchymal transition (EMT) to mesenchymalepithelial transition (MET) states". British Journal of Cancer 110 (2014): 1497-1505.

21. Shaughnessy JO., et al. "Perspectives on the mechanism of action and clinical application of eribulin for metastatic breast cancer". Future Oncology 15.14 (2019): 1641-1653.

22. Funahashi Y., et al. "Eribulin mesylate reduces tumor microenvironment abnormality by vascular remodeling in preclinical human breast cancer models". Cancer Science 105.10 (2014): 1334-1342.

\section{Assets from publication with us}

- Prompt Acknowledgement after receiving the article

- Thorough Double blinded peer review

- Rapid Publication

- Issue of Publication Certificate

- High visibility of your Published work

Website: www.actascientific.com/

Submit Article: www.actascientific.com/submission.php

Email us: editor@actascientific.com

Contact us: +919182824667 